\title{
Next generation sequencing analysis of nine Corynebacterium ulcerans isolates reveals zoonotic transmission and a novel putative diphtheria toxin-encoding pathogenicity island
}

Dominik M Meinel ${ }^{1}$, Gabriele Margos ${ }^{1}$, Regina Konrad ${ }^{1,2}$, Stefan $\mathrm{Krebs}^{3}$, Helmut Blum $^{3}$ and Andreas Sing ${ }^{1,2^{*}}$

\begin{abstract}
Background: Toxigenic Corynebacterium ulcerans can cause a diphtheria-like illness in humans and have been found in domestic animals, which were suspected to serve as reservoirs for a zoonotic transmission. Additionally, toxigenic C. ulcerans were reported to take over the leading role in causing diphtheria in the last years in many industrialized countries.
\end{abstract}

Methods: To gain deeper insights into the tox gene locus and to understand the transmission pathway in detail, we analyzed nine isolates derived from human patients and their domestic animals applying next generation sequencing and comparative genomics.

Results: We provide molecular evidence for zoonotic transmission of $C$. ulcerans in four cases and demonstrate the superior resolution of next generation sequencing compared to multi-locus sequence typing for epidemiologic research. Additionally, we provide evidence that the virulence of $C$. ulcerans can change rapidly by acquisition of novel virulence genes. This mechanism is exemplified by an isolate which acquired a prophage not present in the corresponding isolate from the domestic animal. This prophage contains a putative novel virulence factor, which shares high identity with the RhuM virulence factor from Salmonella enterica but which is unknown in Corynebacteria so far. Furthermore, we identified a putative pathogenicity island for C. ulcerans bearing a diphtheria toxin gene.

Conclusion: The novel putative diphtheria toxin pathogenicity island could provide a new and alternative pathway for Corynebacteria to acquire a functional diphtheria toxin-encoding gene by horizontal gene transfer, distinct from the previously well characterized phage infection model. The novel transmission pathway might explain the unexpectedly high number of toxigenic $C$. ulcerans.

\section{Background}

Diphtheria is the most severe disease attributed to coryneform bacteria [1]. Although Corynebacterium diphtheriae is the classical pathogen described to cause diphtheria, Corynebacterium ulcerans has also been found to cause diphtheria-like illness in humans. Moreover, in recent years cases of human diphtheria caused by C. ulcerans

\footnotetext{
* Correspondence: Andreas.Sing@LGL.Bayern.de

'LGL, Bavarian Health and Food Safety Authority, Oberschleißheim 85764,

Germany

${ }^{2}$ National Consiliary Laboratory on Diphtheria, Oberschleißheim 85764,

Germany

Full list of author information is available at the end of the article
}

seem to outnumber those caused by $C$. diphtheriae in many industrialized countries, including the United Kingdom [2], France [3], the US [4] and Germany [5]. In contrast to $C$. diphtheriae, which to date has been found nearly exclusively in humans, C. ulcerans is often found in domestic animals, which are suspected to serve as reservoirs for possible zoonotic infection. Among those animals were cats, dogs and pigs [6-11]. Additionally, C. ulcerans has also been found in other non-domestic animals, such as cynomolgus macaques [12] and ferrets [13], and in game animals, such as wild boars and roe deer [14]. Although C. ulcerans is considered to be a zoonotic pathogen, molecular indication for zoonotic transmission has 
been found only in four instances, two of them involving dogs $[9,15]$, one a cat $[6]$ and one a pig [10].

Diphtheria is caused by diphtheria toxin (DT)-producing strains of the three Corynebacterium species, $C$. diphtheriae, C. ulcerans and C. pseudotuberculosis. DT is responsible for both the local form of diphtheria, which is characterized by a greyish pseudomembrane at the infection site both in respiratory or cutaneous disease, as well as the systemic symptoms, for example, neurological or cardiac manifestations. DT is a very potent toxin that is able to act on many different types of cells (reviewed in [16]). This Y-shaped protein toxin was shown by X-ray crystallography to consist of three domains [17]. The carboxy-terminal domain of the toxin serves as a receptor, which interacts with the heparin-binding epidermal growth factor precursor on the cell surface $[18,19]$ and is therefore necessary for efficient endocytosis of DT into the cell. The translocator domain forms the middle part of the toxin and is able to integrate into the endosomal membrane upon the $\mathrm{pH}$ change after endocytosis, thereby transferring the amino-terminal, catalytically active part of the toxin into the cytoplasm. The active amino-terminal domain catalyzes the ADP-ribosylation of the translation factor EF-2 with the consumption of NAD and thereby irreversibly inhibits protein synthesis in the cell [20-22]. Remarkably, even a single DT molecule is sufficient to kill a eukaryotic cell [23].

However, not all isolates of $C$. diphtheriae and $C$. ulcerans are toxigenic. It has been reported that infection with a toxigenic phage can cause conversion by integration into the bacterial genome. Noteworthy, the DT encoding tox gene is located at the outer border of the integrated, linearized prophage genome. It is thought that the tox gene was acquired by the phage and might be transferred also to other phages [24]. The expression of the tox gene is controlled by the diphtheria toxin repressor (DtxR), which represses its transcription under high or normal $\mathrm{Fe}^{2+}$ concentrations [25]. DtxR is not encoded by the toxigenic phage, but on the bacterial chromosome [26]. Additionally, DtxR controls not only the toxin gene but also other genes for corynebacterial siderophores, heme oxygenase, and several other proteins [16]. The $\mathrm{Fe}^{2+}$ concentration is usually extremely low in the body fluids of humans or animals and DT is therefore produced by toxigenic strains [16].

Since we and others have registered over recent years many cases of toxigenic C. ulcerans causing diphtherialike disease in humans, we aimed to analyze the toxigenic conversion of C. ulcerans. Resequencing data from nine $C$. ulcerans strains which were isolated from four human patients and their domestic animals showed that the bacteria strains were transmitted zoonotically. Moreover, we found that the pathogenic potential of $C$. ulcerans can change very rapidly due to infection by a phage containing a novel virulence gene, which was firstly described in Salmonella, and we also describe a novel DT-encoding putative pathogenicity island (PAI) which differs completely from the so far known toxigenic prophages of Corynebacteria.

\section{Methods}

\section{Culture of bacteria and DNA isolation}

C. ulcerans isolates were grown in liquid culture using Thioglycolat-Bouillon $\left(37^{\circ} \mathrm{C}\right.$ aerobic conditions). The $\mathrm{C}$. ulcerans isolates were taken from the German Consiliary Laboratory on Diphtheria (NCLoD) isolate collection. The investigations were performed as part of public health outbreak investigations. Therefore, additional ethical approval was not required. Isolate species were determined by matrix-assisted laser desorption/ionization (MALDI)-time of flight (TOF) mass spectrometry and/ or biochemical testing and the isolates were tested for toxigenicity by DT-PCR as described in [27]. The Elek test for DT expression was performed according to [28]. For next generation sequencing (NGS), $20 \mathrm{ml} \mathrm{C.} \mathrm{ulcer-}$ ans culture was harvested by centrifugation and DNA was extracted after lysozyme digestion at $37^{\circ} \mathrm{C}$ for $15 \mathrm{mi}-$ nutes using a Maxwell 16 DNA extraction device (Promega, Mannheim, Germany). Bacteria were treated with lysis buffer containing Proteinase $\mathrm{K}$ and RNase for $2 \mathrm{~h}$ at $65^{\circ} \mathrm{C}$ and DNA purification was performed as described by the manufacturer.

\section{Genome sequencing, draft assembly and analysis}

After quality control of the DNA, a tagmentation library was generated as described by the manufacturer (NexteraXT kit, Illumina, San Diego, CA, USA). The genomes were sequenced as multiplexed samples using a $2 \times 250$ bp V2 reaction kit on an Illumina MiSeq instrument reaching an average coverage of approximately 50fold for all isolates. After quality control of the raw data, the reads were adapter clipped and quality trimmed and downstream analysis was carried out using a local instance of Galaxy [29-31]. We used SOAP denovo (v.1.0.0) for assembly of the genome [32] and BWA for Illumina (v.1.2.3) [33] for mapping the reads to the reference genome $C$. ulcerans 809 [34]. The mapping was refined using SRMA (v.0.2.5) [35]. SNPs were determined for the sequenced isolates and the published $C$. ulcerans genomes using VarScan (v.2.3.2) [36] and R (v.3.0.3, CRAN) [37]. The used $\mathrm{R}$ scripts are available upon request. Since we employed the C. ulcerans 809 genome as a reference, which carries a prophage in its genome, we excluded the region harboring the prophage from the analysis [34].

As we aimed to compare our resequencing data with the published finished genomes without losing quality information in our resequencing data, we only used SNPs which could be unambiguously identified in our sequenced dataset. This implies that the regions not covered by our 
re-sequencing are not included in the analysis. To prevent acceptance of false negative SNPs, we firstly determined a set of SNPs that could be called with very high quality (minimum coverage of 20 reads and at least $90 \%$ variant frequency) in at least one of our samples and compiled a list of trustworthy SNP positions in our sequenced genomes. In the next step, we used this list to determine if these SNPs are also present in the other isolates - that is, we analyzed all those positions of the trustworthy SNPs in all isolates by allowing identification of the presence of SNPs at the given position with lower quality criteria. The lower quality criteria were minimum coverage of two-fold with at least a variant frequency of $>50 \%$.

The first step ensures that we only consider positions within the genomes with reliable SNPs. The second step ensures that, upon identification of a SNP at a certain position in one isolate, the remaining isolates are not false negatives due to too little coverage - that is, the quality of SNP calling - at the corresponding position.

For the detailed analysis of matched isolates (isolates within a pair), we manually curated the intra-pair SNPs; that is, we excluded from both isolates SNPs that we were not able to correctly determine in one of the two strains due to missing data at the corresponding genomic position. Therefore, we deleted a SNP from the manually corrected list of an isolate if it was not possible to determine in the matched isolate whether there is a SNP or not at the corresponding position. Thereby we avoided false negative SNPs (that is, negative detection due to missing data), which would lead to possibly spurious differences between two isolates when comparing them. We did not perform manual curation for the inter-pair SNPs, since random inspection showed that only a very minor fraction of the SNPs in this category was due to coverage problems (less than 3 out of 1,000 SNPs). This is most likely caused by the fact that the critical positions where only one of the isolates has sufficient sequencing coverage are very small compared with the remaining genome and form an approximately constant false negative SNP background level, which only reaches a considerable fraction for a small number of real SNPs. For calculation of the phylogenetic trees, we exported the SNPs, and concatenated and constructed the phylogeny (neighbor joining) using MEGA 6.0 [38]. BRIG [39], Artemis [40] and IGV [41] were used for visualization of the data. Multi-locus sequence typing (MLST) SNP data for $\operatorname{atp} A, \operatorname{dnaE}$, dnaK, fus $A$, leuA, odhA and rpoB were extracted from the NGS dataset.

$x$ Base was used for the annotation of the draft genome [42]. Contigs were sorted using Mauve [43] and concatenated using the genomic sequence of C. ulcerans 809 [34] as reference. xBase uses Glimmer for gene prediction [44], and tRNAScan-SE [45] and RNAmmer [46] for prediction of tRNAs and rRNAs. BLAST was used for annotation of the predicted proteins [47]. Prophages were searched using PHAST [48]. Therefore, we sorted our de novo assembled contigs and the contigs of FRC58 [49] versus the reference genome of C. ulcerans 809 and analyzed the concatenated sequences with PHAST. Annotated proteins were further analyzed with BLAST, HHPred [50] and InterPro [51] Multiple alignments were calculated with Clustal Omega [52] and visualized with Jalview [53].

\section{Next generation sequencing data}

All sequencing data are available from the Sequence Read Archive [54] under experiment accession number SRX740276. The annotated region of the putative PAI is available at GenBank (KP019622).

\section{Results}

Toxigenic $C$. ulcerans outnumber toxigenic $C$. diphtheriae Wagner et al. [2] found that toxigenic C. ulcerans infections outnumber toxigenic $C$. diphtheriae infections in diphtheria patients in the United Kingdom. We wondered whether this phenomenon could be due to a higher proportion of toxigenic versus non-toxigenic $C$. ulcerans compared with the proportion of toxigenic versus non-toxigenic $C$. diphtheriae. Therefore, we analyzed the database of the NCLoD at the Bavarian Health and Food Safety Authority. The isolates analyzed here were sent for differentiation to the NCLoD by several clinical microbiology laboratories and as a caveat might not be representative of the whole Corynebacterium population in Germany and several of the Corynebacteria were isolated from animals. Among the $103 \mathrm{C}$. diphtheriae isolates sent to the NCLoD between 2010 and 2013, 13 (12.4\%) were tox-positive (Figure 1). In contrast, a much higher proportion of $C$. ulcerans carried the tox gene (33/47; 70.2\%). This might indicate that C. ulcerans acquires the toxin gene more easily or that the suspected zoonotic transmission might favor toxigenic conversion of C. ulcerans.

\section{Comparative genomics reveals zoonotic transmission of C. ulcerans}

To address the question of whether C. ulcerans is a zoonotic pathogen, we analyzed nine toxigenic C. ulcerans isolates by NGS. The isolates form three pairs and one triplet. Within each pair we analyzed the C. ulcerans isolate from a human patient and one isolate from their domestic animals (for a description of the pairs see Table 1). In one case, a patient owned two cats, which were positive for $C$. ulcerans; therefore, we included an additional group, a triplet, consisting of isolates from the patient and the two cats ('pair B'). We performed resequencing with an Illumina MiSeq sequencer, and analyzed the obtained genomic information for SNPs using C. ulcerans 809 (GenBank CP002790) as reference 


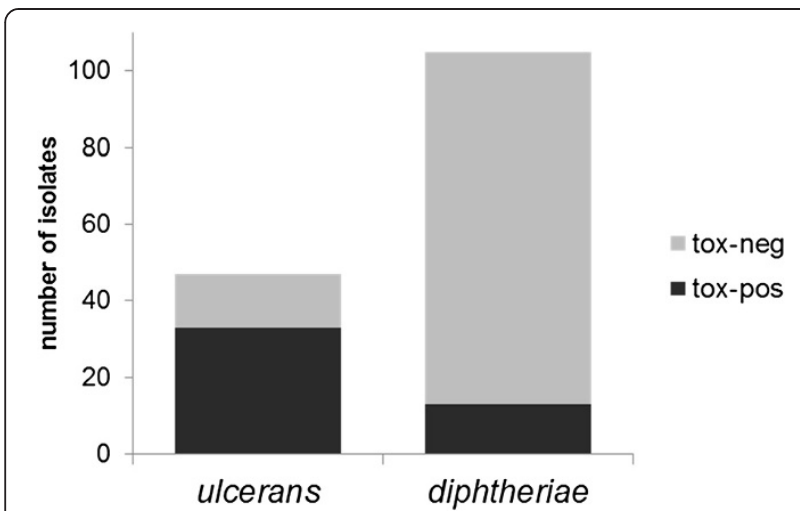

Figure 1 Toxigenic and non-toxigenic $C$. ulcerans and $C$. diphtheriae isolates from 2011 to 2013. Corynebacterium isolates sent to the NCLoD. Species and toxigenicity of the isolates were determined using MALDI mass spectroscopy and PCR, respectively. The isolates are derived from human patients and animals.

genome [34]. The average coverage per genome was approximately 50-fold. Additionally, we also included other published C. ulcerans genomes from Brazil [34] and Japan [24] and a draft genome from France [49] for comparative genome and phylogenetic analysis.

Interestingly, NGS revealed that $C$. ulcerans isolates from different groups varied among each other at a substantial number of SNPs (5,000 to 20,000 SNPs; Table 2) throughout the whole genome, while the isolates within a pair only showed differences at single SNPs (Table 2). SNPs found within the same group were manually curated to exclude false positive SNPs (see Methods section for details). The intra-group differences were unexpectedly small and strongly indicate that the isolates within the same group originate from a common precursor. Due to the very low number of SNPs within the groups (0 to 2 SNPs), we also conclude that zoonotic transmission took place within each group very recently (Figure 2). Interestingly, three out of four pairs from Germany and a published French draft genome of a C. ulcerans isolate cluster together, as also depicted by the phylogenetic analysis using the genome-wide data (Figure 2A). This result was reproducible with different phylogenetic analysis algorithms (neighbor joining, maximum parsimony, maximum likelihood; Figure S1 in Additional file 1), suggesting a European genotype for C. ulcerans which is different from the genotypes described from South America [34] and Asia [24]. Furthermore, we found that one pair of our collection did not cluster with the other pairs but with the genome of an isolate from Japan (Figure 2A). Remarkably in this context, our isolates clustering with the Japanese isolate (C. ulcerans 0102) shared one prophage with $C$. ulcerans 0102 which was shown to carry the DT encoding tox gene, but lacked the two other prophages identified in the C. ulcerans 0102 genome. Overall, we showed using NGS a zoonotic relationship in all four analyzed pairs of $C$. ulcerans isolated from humans and their domestic animals.

\section{Genome resequencing adds critical information to MLST}

In a next step we asked whether MLST is comparable to NGS resequencing for, for example, outbreak analysis. Therefore, we compared MLST with NGS (Figure 2B): as expected by the much smaller genomic regions analyzed in MLST, we found only very few SNPs in the analyzed strains. The number of SNPs in the MLST analysis was not sufficient to discriminate pairs A and D from each other. Nonetheless, MLST recapitulated the clustering of pairs A, B and D near to the isolate from France and also found a cluster with the Japanese isolate and pair C. Noteworthy, phylogenetic analysis of the MLST data with different algorithms did not robustly reproduce the phylogenetic relationship, as indicated by low bootstrapping values (Figure 2; Figure S1 in Additional file 1). Thus, we conclude that MLST is still a helpful, fast and cost-effective tool for rough phylogenetic analysis, but NGS resequencing is superior fordetailed outbreak analysis and provides the resolution needed for in-depth understanding of transmission pathways.

\section{Table 1 Isolates used for sequencing in this study}

\begin{tabular}{|c|c|c|c|c|c|c|}
\hline Pair & Identifier & Host & Symptoms & $G+C$ content & Assembled contigs & CDS \\
\hline A & KL126 & Human & Throat diphtheria-like disease & $53 \%$ & 41 & 2,264 \\
\hline$A$ & $08-1143$ & Pig & Asymptomatic & $53 \%$ & 33 & 2,274 \\
\hline B & KL246 & Human & Throat diphtheria-like disease & $53 \%$ & 30 & 2,270 \\
\hline B & KL251 & Cat & Asymptomatic & $53 \%$ & 30 & 2,272 \\
\hline B & KL252 & Cat & Asymptomatic & $53 \%$ & 34 & 2,270 \\
\hline C & KL315 & Human & Ulcus, lower leg & $53 \%$ & 34 & 2,276 \\
\hline C & KL318 & Dog & Asymptomatic & $53 \%$ & 31 & 2,269 \\
\hline D & KL387 & Human & Wound & $53 \%$ & 32 & 2,231 \\
\hline $\mathrm{D}$ & KL392 & Cat & Asymptomatic & $53 \%$ & 38 & 2,304 \\
\hline
\end{tabular}

Isolates analyzed in this study were derived from the NCLoD at the Bavarian Health and Food Safety Authority. To our knowledge, the wounds of the patients from which the $C$. ulcerans were isolated were not caused by the animals. Additionally, given are the number of assembled contigs, their average $G+C$ content and the number of predicted protein coding sequences (CDSs). 
Table 2 SNPs found in the Corynebacterium ulcerans isolates

\begin{tabular}{lllllllllllll}
\hline & $\mathbf{0 8 - 1 1 4 3}$ & $\mathbf{K L 1 2 6}$ & $\mathbf{K L 2 4 6}$ & $\mathbf{K L 2 5 1}$ & $\mathbf{K L 2 5 2}$ & $\mathbf{K L 3 1 5}$ & $\mathbf{K L 3 1 8}$ & $\mathbf{K L 3 8 7}$ & KL392 & FRC58 & $\mathbf{1 0 2}$ & BR-AD22 \\
\hline $\mathbf{0 8 - 1 1 4 3}$ & 0 & $2(35)$ & 7,293 & 7,304 & 7,300 & 21,271 & 21,258 & 7,330 & 7,337 & 7,585 & 20,538 & 17,757 \\
$\mathbf{K L 1 2 6}$ & $2(35)$ & 0 & 7,291 & 7,290 & 7,290 & 21,263 & 21,256 & 7,323 & 7,326 & 7,579 & 20,536 & 17,758 \\
$\mathbf{K L 2 4 6}$ & 7,293 & 7,291 & 0 & $0(46)$ & $1(32)$ & 17,269 & 17,256 & 5,239 & 5,254 & 9,285 & 16,773 & 16,619 \\
$\mathbf{K L 2 5 1}$ & 7,304 & 7,290 & $0(46)$ & 0 & $1(51)$ & 17,281 & 17,266 & 5,259 & 5,270 & 9,294 & 16,784 & 16,635 \\
KL252 & 7,300 & 7,290 & $1(32)$ & $1(51)$ & 0 & 17,267 & 17,254 & 5,222 & 5,245 & 9,270 & 16,763 & 16,608 \\
KL315 & 21,271 & 21,263 & 17,269 & 17,281 & 17,267 & 0 & $0(96)$ & 16,602 & 16,608 & 16,603 & 1,297 & 12,670 \\
KL318 & 21,258 & 21,256 & 17,256 & 17,266 & 17,254 & $0(96)$ & 0 & 16,591 & 16,589 & 16,585 & 1,280 & 12,659 \\
KL387 & 7,330 & 7,323 & 5,239 & 5,259 & 5,222 & 16,602 & 16,591 & 0 & $2(37)$ & 9,521 & 16,079 & 16,034 \\
KL392 & 7,337 & 7,326 & 5,254 & 5,270 & 5,245 & 16,608 & 16,589 & $2(37)$ & 0 & 9,550 & 16,087 & 16,050 \\
FRC58 & 7,585 & 7,579 & 9,285 & 9,294 & 9,270 & 16,603 & 16,585 & 9,521 & 9,550 & 0 & 16,316 & 16,166 \\
$\mathbf{1 0 2}$ & 20,538 & 20,536 & 16,773 & 16,784 & 16,763 & 1,297 & 1,280 & 16,079 & 16,087 & 16,316 & 0 \\
BR-AD22 & 17,757 & 17,758 & 16,619 & 16,635 & 16,608 & 12,670 & 12,659 & 16,034 & 16,050 & 16,166 & 12,122 & 0 \\
\hline
\end{tabular}

SNPs of the resequenced isolates show that only very minor differences are detectable within each group of isolates. Numbers in parentheses represent the number of SNPs originally given by the SNP calling pipeline. The number in front is the number of SNPs remaining after manual curation as described in the Methods section to avoid faulty SNP calling.

\section{C. ulcerans typically carries one or more prophages}

Infection of $C$. diphtheriae or C. ulcerans with a tox-carrying phage can lead to toxigenic conversion of the bacterium. Therefore, we surveyed how common prophage insertions are in C. ulcerans genomes. We sorted the de novo assembled contigs versus $C$. ulcerans 809 as reference genome and analyzed the genome for putative prophages using the PHAST algorithm [48]. We found putative prophages in most of the isolates which were sequenced in this study and also in the published $C$. ulcerans genomes (summarized in Table 3). As mentioned above, we detected the same toxigenic phage as in C. ulcerans 0102 in both isolates of pair C [24]. Interestingly, the other two prophages found in C. ulcerans 0102 were not present in pair $C$, isolated from a patient and a dog from Germany. In summary, we found in all isolates, except for pair A, between one and four putative prophages, suggesting that phage infection is commonly occurring in C. ulcerans (Table 3 ).

In a next step we compared the putative phage content of the individual isolates forming a human-animal pair and found that the predicted prophage content was nearly identical. We found only that KL387 and KL392 (pair D) differ in their putative prophage content (Figure 3A), although the SNP analysis of the humananimal isolate pair showed only very minor differences (two verified SNPs in approximately $2.5 \mathrm{Mb}$ ). This finding strongly indicates that both isolates originate from the same parental $C$. ulcerans strain and the very low number of detected SNPs argues for a recent event of phage integration, likely because there was insufficient time to acquire new SNPs in the meantime. The additional putative prophage in KL387 is integrated just downstream of the tRNA-Thr locus (anticodon: CGT) and is flanked by an 85 bp direct repeat with $100 \%$ identity (426.686-426.771 and 459378-459463 bp in KL387). One of the two repeats is, as expected, also present in KL392. The integration near a tRNA locus and the duplication of a short genomic region flanking the integration region of the prophage are typical features found at prophage integration sites in many bacteria [58]. Additionally, the local GC content in the putative prophage region of KL387 is considerably lower than the GC content of the genomic region surrounding the putative prophage. This is typically found at prophage integration sites [58] and strongly suggests an event of horizontal gene transfer in this region.

Furthermore, closer analysis of the predicted genes in the putative prophage revealed, for all predicted sequences, known phage homologues or proteins associated with putative prophages from other bacteria (Figure 3B). Excitingly, we found one predicted protein that shows high identity to the Fic toxin of Bacillus massiliosenegalensis and to the RhuM virulence factor from the Salmonella enterica pathogenicity island 3 (SPI-3). RhuM (NP_462654) and the predicted phage protein shared $42.3 \%$ identity and $58.3 \%$ similarity (Figure 3C). It was shown that RhuM inactivation leads to highly reduced virulence of Salmonella and to a lower mortality rate after $S$. enterica infection in the Caenorhabditis elegans model [59]; however, no clear molecular function for this protein is known. Therefore, increased virulence of KL387 versus KL392 caused by the integration of the phage remains to be shown. We hypothesize, however, that the conversion by a virulence factor- or toxin-carrying phage of C. ulcerans can take place very rapidly and might change the virulence of the strain even within short periods of times - for example, even within a single zoonotic transmission event. 

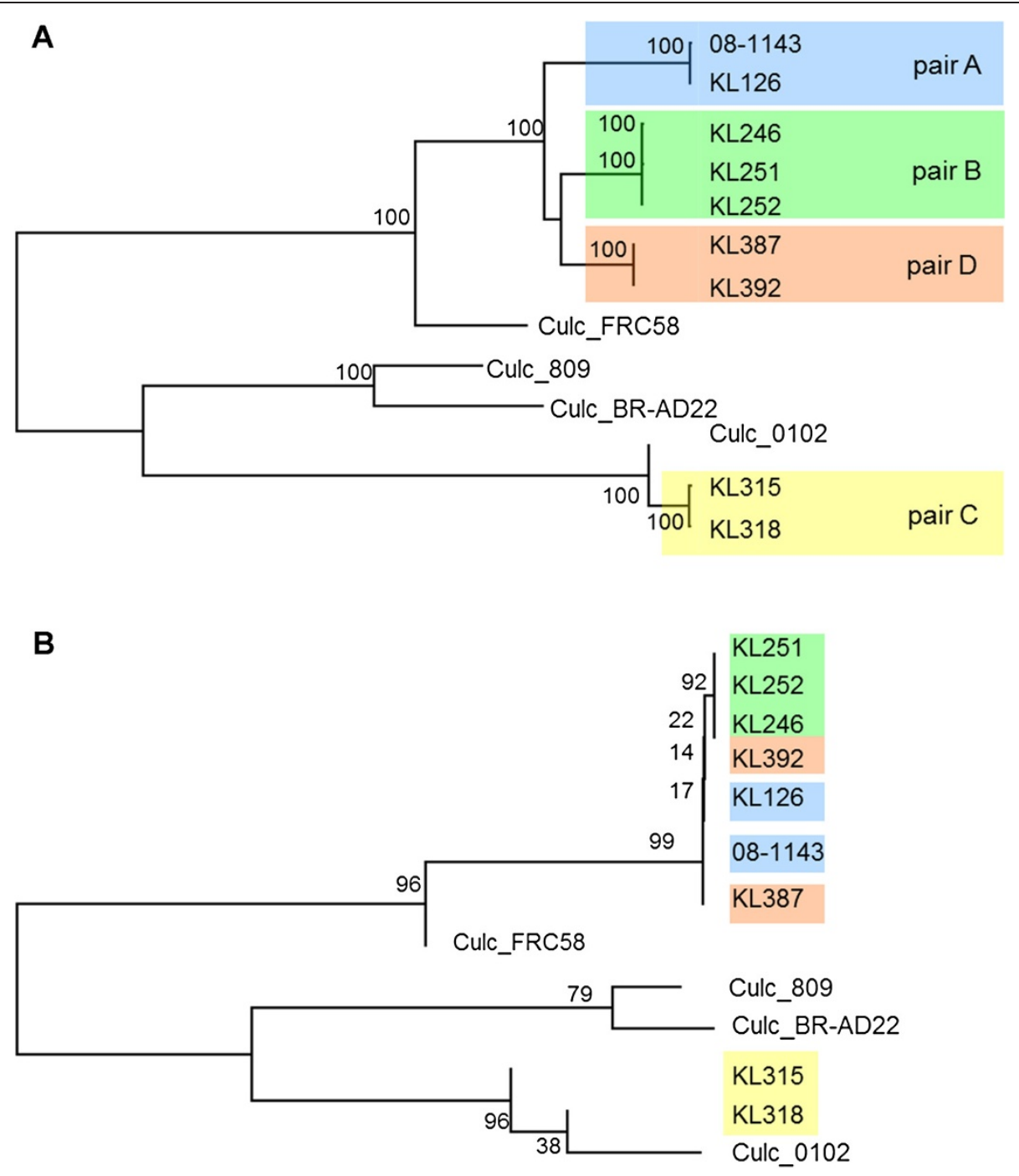

Figure 2 Resequencing reveals zoonotic transmission of $C$. ulcerans and improves resolution in phylogeny compared with multi-locus sequence typing. (A) Whole genome sequence phylogenetic analysis of the C. ulcerans isolates. The evolutionary history was inferred using the neighborhood joining method [55]. The percentage of replicate trees in which the associated taxa clustered together in the bootstrap test (100 replicates) are shown next to the branches [56]. The isolates within the pairs are indistinguishable from each other in the dendrogram, indicating very close relationship or even identity, while the isolates of other pairs are clearly separated (B) Phylogenetic analysis for seven MLST loci as in [57]. The phylogenetic analysis was conducted as in Figure 2A. KL251, KL252, KL392, KL126, 08-1143 and KL 387 fall together into one cluster which offers no information on the substructure (bootstrap values 14 to 19), showing that the resolution of MLST is not high enough to sort the isolates into the three pairs as in Figure $2 \mathrm{~A}$.

\section{A novel, putative diphtheria toxin-encoding pathogenicity} island in C. ulcerans

In the isolates KL315 and KL318 (forming pair C) the DTencoding tox genes were located in a predicted prophage region which exhibits very high identity to the toxigenic prophage of C. ulcerans 0102 (99\% identity) [24]. Conversion of a non-toxigenic to a toxigenic bacterium by prophage integration is well described for $C$. diphtheriae and is also assumed to take place in C. ulcerans.

Additionally, we found in seven out of nine toxigenic isolates a novel, unknown and putative PAI harboring the DT encoding gene (Figure 4A): the novel, putative PAI was present in KL126, 08-1143, KL246, KL251, KL252, KL387, and KL392 and is in all seven strains located at the same genomic site, just downstream of the tRNA-Arg (anticodon: ACG). Interestingly, this locus is known to be targeted by many events of horizontal gene transfer: the toxigenic prophages from C. ulcerans 0102 [24], KL315 and KL318 are integrated into this locus. Additionally, a putative virulence factor has been found at this genomic position in C. ulcerans 809 and was hypothesized to be a ribosome binding protein that shares 
Table 3 C. ulcerans genome usually encode several Prophages

\begin{tabular}{|c|c|c|c|c|c|c|c|c|c|c|}
\hline Isolate & $\begin{array}{l}\text { Prophage } \\
\text { identifier }\end{array}$ & $\begin{array}{l}\text { Prophage } \\
\text { size }(\mathbf{k b})\end{array}$ & Possible derivate of & $\begin{array}{l}\text { Virulence } \\
\text { factor }\end{array}$ & CDS & $\begin{array}{l}\mathrm{G}+\mathrm{C} \\
\text { content }\end{array}$ & Position (bp) & Att & $\begin{array}{l}\text { Identity with } \\
\text { other isolate of pair }\end{array}$ & Reference \\
\hline KL246 & 1 & 18.2 & Corynephage_BFK20 & & 18 & $52 \%$ & $440890-459165$ & ND & $100 \%$ & \\
\hline KL251 & 1 & 18.2 & Corynephage_BFK20 & & 18 & $52 \%$ & $441088-459363$ & ND & $100 \%$ & \\
\hline KL252 & I & 18.2 & Corynephage_BFK20 & & 18 & $52 \%$ & $436472-454747$ & ND & $100 \%$ & \\
\hline KL315 & I & 39.3 & DCULC0102-I & $\begin{array}{l}\text { Diphtheria } \\
\text { toxin }\end{array}$ & 28 & $55 \%$ & $142798-182119$ & Yes & $100 \%$ & \\
\hline KL318 & I & 39.0 & DCULC0102-I & $\begin{array}{l}\text { Diphtheria } \\
\text { toxin }\end{array}$ & 27 & $55 \%$ & 291509-33059 & Yes & $100 \%$ & \\
\hline \multirow[t]{3}{*}{ KL387 } & I & 18.2 & Corynephage_BFK20 & $\begin{array}{l}\text { Putative } \\
\text { RhuM }\end{array}$ & 18 & $52 \%$ & $438665-456940$ & ND & ND & \\
\hline & $\|$ & 39.8 & $\begin{array}{l}\text { Rhodococcus } \\
\text { phage REQ2 }\end{array}$ & & 50 & $54 \%$ & 1898856-1938678 & Yes & $100 \%$ & \\
\hline & III & 10.4 & $\begin{array}{l}\text { Mycobacterium } \\
\text { phage Fishburne }\end{array}$ & & 17 & $52 \%$ & $2527900-2538480$ & ND & $92 \%$ & \\
\hline \multirow[t]{2}{*}{ KL392 } & I & 42.1 & $\begin{array}{l}\text { Rhodococcus } \\
\text { phage REQ2 }\end{array}$ & & 54 & $55 \%$ & $1858755-1900873$ & Yes & $100 \%$ & \\
\hline & $\|$ & 10.4 & $\begin{array}{l}\text { Mycobacterium } \\
\text { phage Fishburne }\end{array}$ & & 17 & $52 \%$ & $2505077-2515476$ & ND & $92 \%$ & \\
\hline \multirow[t]{3}{*}{102} & I & 38.3 & DCULC0102-I & $\begin{array}{l}\text { Diphtheria } \\
\text { toxin }\end{array}$ & 26 & $54 \%$ & $168523-206883$ & Yes & & [24] \\
\hline & $\|$ & 21.4 & DCULC0102-II & & 18 & $52 \%$ & $536771-558192$ & ND & & [24] \\
\hline & III & 39.4 & DCULC0102-III & & 22 & $57 \%$ & $1377963-1417370$ & Yes & & [24] \\
\hline \multirow[t]{4}{*}{ BR-AD22 } & I & 42.0 & DCULC22-I & & 42 & $53 \%$ & 1299138-1338708 & ND & & [34] \\
\hline & $\|$ & 44.9 & DCULC22-II & & 60 & $55 \%$ & 1853009-1877311 & Yes & & [34] \\
\hline & III & 14.0 & DCULC22-III & & 19 & $57 \%$ & 1963728-1986514 & Yes & & [34] \\
\hline & IV & 41.0 & DCULC22-IV & & 53 & $54 \%$ & 2134999-2156991 & Yes & & [34] \\
\hline 809 & I & 41.4 & DCULC809-I & & 45 & $53 \%$ & $1295507-1335046$ & ND & & [34] \\
\hline FRC58 & I & 29.4 & $\begin{array}{l}\text { Mycobacterium } \\
\text { phage Fishburne }\end{array}$ & & 52 & $53 \%$ & 2493492-2522907 & Yes & & [49] \\
\hline
\end{tabular}

Prophages are as annotated by PHAST [48] or in [24,34]. Given are the isolate and prophage identifiers, the predicted prophage size and the name of the prophage sharing the highest similarity with the predicted prophage. The shared identity of the prophage with the corresponding pair isolate is given as a percentage. The prophage KL387-III was not predicted by PHAST, most likely due to a contig boundary. However, the alignment with KL392-III clearly identified the prophage region KL387-III with $100 \%$ identity. KL387-I was blasted versus the whole genome data of KL392 but no similarity was identified, arguing against a false negative detection of this prophage in the KL392 genome. Att.: predicted attachment site of the phage. CDS, coding sequence.

high similarity with the Shiga toxin [34], which we were unable to detect it in our isolates. Furthermore, this conserved tRNA site is described in $C$. diphtheriae as an integration site for toxigenic and other prophages [60-62] and it seems that this integration hot spot in the Corynebacterium genome is highly conserved, as it has been reported that phage integration can take place at this tRNA locus in at least three different Corynebacterium species [63].

We initially identified the novel, putative toxigenic PAI by analysis of the local GC content, which is strongly reduced in a region around the DT gene. The putative PAI localizes just downstream of a tRNA-Arg (anticodon: ACG) and parts of the tRNA have been duplicated leading to a predicted pseudo-tRNA at the 3' end of the PAI, with a perfect 100 bp directed repeat. Comparison with other available genome data and analysis of the duplicated region within the putative PAI suggest a size of
7,571 bp for the PAI. The GC content of approximately $48 \%$ compared with an average GC content of approximately $53 \%$ for the whole genome of C. ulcerans together with the $100 \mathrm{bp}$ directed repeat strongly indicate horizontal gene transfer [66]. The novel C. ulcerans PAI was predicted to contain eight proteins. Most interestingly, among these we found the DT precursor (Figure 4B). It is located to the 3 ' end of the PAI just upstream of the pseudo-tRNA. The tox gene is $>99 \%$ identical to the alleles described for C. ulcerans [67]. We found for several of the isolates (for example, KL126 and 252) that the DT was expressed in sufficient amounts to result in positive signals in the Elek test, indicating functional DT expression. Additionally, a protein of the PAI was predicted to be a transposase and the adjacent gene was predicted to encode a protein containing a homeodomain-like (HO-like) domain with a helix-turn-helix (HTH)-like motif. 


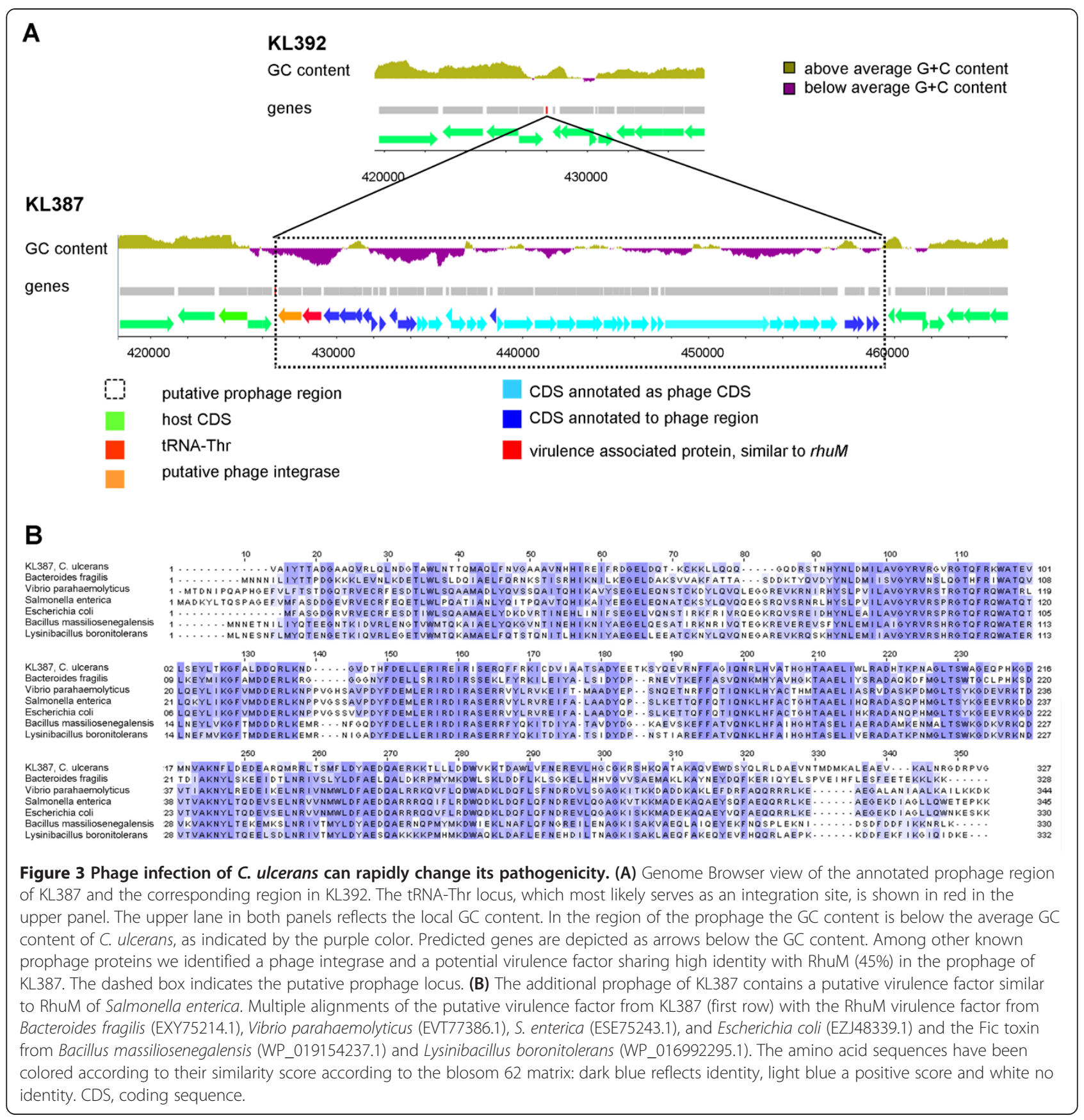

This protein shares high similarity with known insertion elements from other Corynebacterium species. Bioinformatics analyses suggest that it might serve as a transcriptional regulator by sequence-specific DNA binding via its HO-like domain (Figure 4B). Furthermore, we identified a putative integrase/Tyr-recombinase and a putative transcription regulator containing an $\mathrm{HTH}$-like domain (Figure 4B). HTH motifs are known to bind DNA in a sequence-specific manner. In addition to the HTH-like domain, this protein also carries a DUF955 domain which has no known function but is suspected to be catalytically active, since the $\mathrm{H}-\mathrm{E}-\mathrm{X}-\mathrm{X}-\mathrm{H}$ motif might bind metal ions and serve as a hydrolase (Figure 4B). Remarkably, among the eight predicted polypeptides of this novel, putative PAI we found a second putative protein of unknown function carrying a similar DUF955 domain (Figure 4B). This novel, putative PAI is highly conserved within the seven isolates. We only detected one SNP within this PAI within all seven isolates, showing its high conservation. 


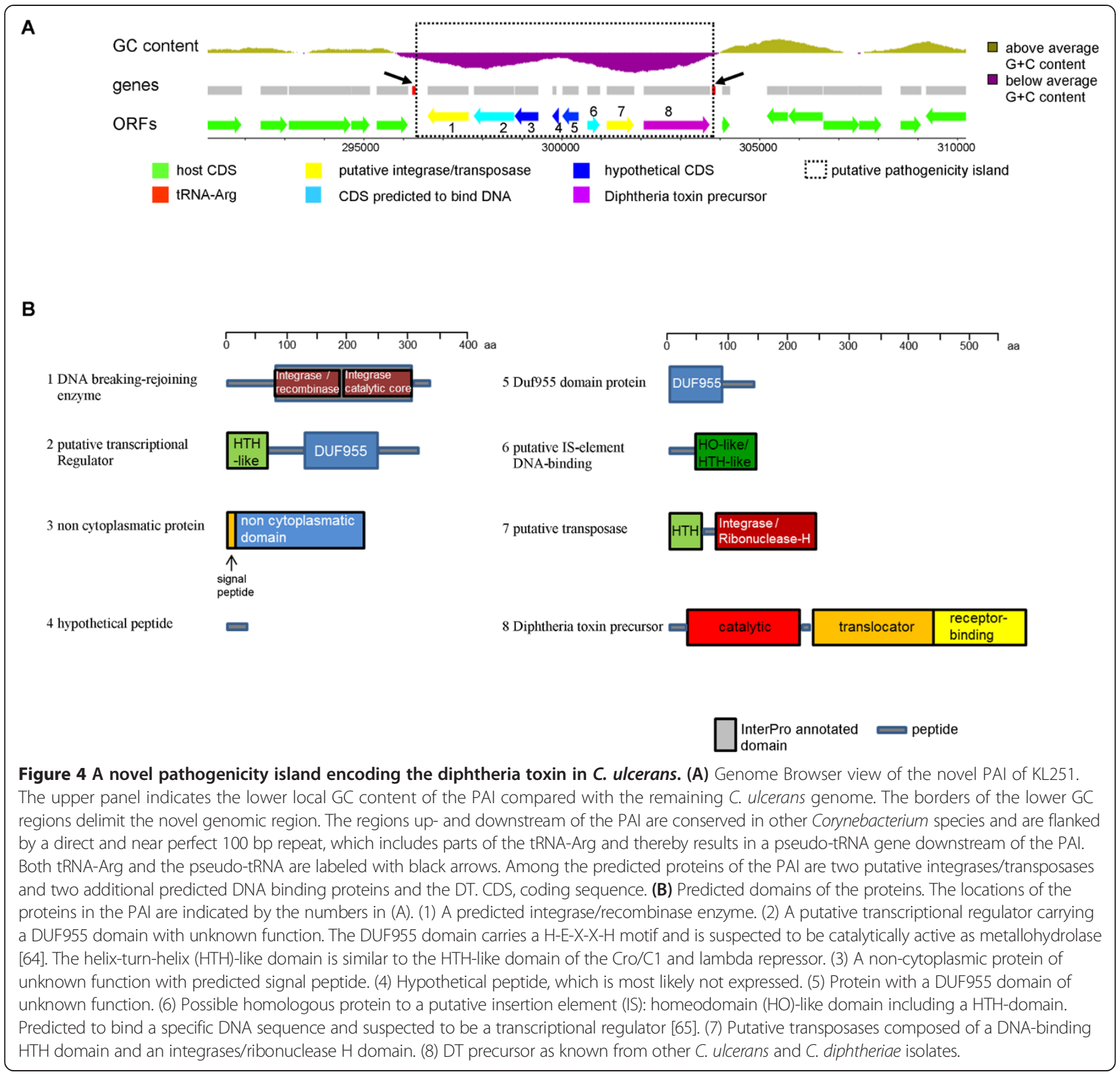

\section{Discussion}

The presented study of nine C. ulcerans draft genomes demonstrates for the first time the zoonotic transmission of toxigenic C. ulcerans on a molecular level, which was previously predicted by sequence data of single gene fragments and ribotyping. We report that pairs of patient and companion/domestic animal isolates of $C$. ulcerans show no or only very few differences in their genome-wide SNP profiles, while the isolates obtained from different patients and/or animals show many more differences. This proves that $C$. ulcerans undergoes zoonotic transmission between animals and humans. Additionally, the results illustrate that analysis by NGS improves the toolkit for phylogenetic and epidemiological studies, by adding more detailed information, more resolution and more robust discrimination between closely related isolates.

Moreover, our data show that C. ulcerans isolates often carry one or more prophages which are able to modify pathogenicity of the bacteria. Interestingly, we found that even within the pair of isolates derived from a patient (KL387) and their cat (KL392), phage integration can take place. Even though both isolates do not differ from each other in their SNP profiles (we only detected two SNPs) and indels, we found that the isolate from the human patient carried a prophage. Since we could not detect any remnants or duplicated sequences in KL392 in proximity to the tRNA-Thr locus, where the 
prophage is integrated in KL387, we suppose that the prophage was integrated into KL387 rather than excised from KL392. In addition, we found a putative virulence factor among the predicted proteins of the prophage. This protein shared high identity with RhuM, a protein from $S$. enterica. It was shown in a C. elegans model to be important for epithelial cell invasion of $S$. enterica [59]. A molecular function for RhuM in S. enterica is not known, but sequence analysis points towards DNAbinding activity [68]. Additionally, deletion of rhuM reduced the fraction of killed C. elegans upon Salmonella infection by approximately half [59]. We did not assay for changed pathogenicity of the isolates carrying the rhuM homologous gene but it would be very interesting to know whether rhuM expression leads also to higher virulence of $C$. ulcerans similar to $S$. enterica, using an C. ulcerans infection model [69]. Nevertheless, here we provide evidence that prophages can be taken up and integrated very rapidly into the $C$. ulcerans genome, in the reported case even within one zoonotic transmission event. As a consequence, this might lead to a change in virulence and pathogenicity of C. ulcerans. We showed that NGS analysis is able to identify such novel gene acquisitions and other genomic modifications in bacteria very efficiently. This strongly underlines that, for detailed and comprehensive epidemiologic surveillance and monitoring of pathogens, NGS analysis represents a very effective tool to identify emerging critical changes in the virulence of bacteria.

Furthermore, considering the higher proportion of toxigenic versus non-toxigenic $C$. ulcerans compared with $C$. diphtheriae, we found that seven out of nine analyzed C. ulcerans isolates carried a putative PAI which is completely different to the known prophages encoding DT. To our knowledge no case of a Corynebacterium carrying a DT gene which is not located in a prophage region has been described to date. There are indications that the putative PAI might be inserted by horizontal gene transfer into a recombination hot spot in the Corynebacterium genome. This recombination hotspot has been described for several Corynebacterium species [63]. Firstly, we found that the GC content of the PAI region differed from the remaining genome. Secondly, we found putative integrases/recombinases and terminal direct repeats (Figure 4A), duplicating parts of the tRNA-Arg adjacent to the putative PAI. Since this genomic site is highly conserved in several Corynebacterium species and is known to serve for other integration events as a target/ attachment site (for example, for prophages), it would be interesting to analyze other toxigenic Corynebacterium species to see if they also contain this novel, putative PAI or a similar insert. Alternatively, this PAI could be specific to C. ulcerans and might, therefore, be the reason for the higher proportion of toxigenic C. ulcerans.
The finding of the novel tox gene encoding a putative PAI leads to the very important question for future research of whether the whole identified PAI forms a functional unit. One hypothesis is that the PAI is a large 'hybrid transposon', encoding a transposase and other recombination enzymes, which targets the tRNA-Arg recombination site. Containing the DT gene, it may represent an additional virulence factor which can spread by horizontal gene transfer. Another possibility would be that the PAI originated by several events. For instance, it can be speculated that several insertion elements, one of which carried the tox gene, integrated into this genomic site. However, since we found seven identical PAIs in nine toxigenic isolates, which differed to a larger extent in the remaining genome, we favor the hypothesis that the putative PAI itself might be a genomic element which can be transferred horizontally between $C$. ulcerans. If the PAI developed in several strains in parallel, we would expect less conservation and more SNPs and most likely different compositions for it between the different pairs of isolates. The idea of horizontal transfer is supported by the finding that the PAI contains genes for two integrase/transposase-like proteins and at least two additional predicted DNA-binding proteins, which share similarity with proteins involved in horizontal gene transfer (phages/insertion elements). Such proteins would be expected in a putative 'hybrid transposon' which could insert to a target site via the site-specific binding/action of its encoded proteins. An efficient horizontal transfer mechanism could also well explain why such a large fraction of the isolates are toxigenic and the high conservation of the novel PAI.

Furthermore, it is an interesting point to speculate why the proportion of toxigenic and non-toxigenic strains among C. ulcerans outnumbers that of $\mathrm{C}$. diphtheriae in our strain collection. A possible hypothesis is that this PAI is specific for $C$. ulcerans and that it spreads much more efficiently than the toxigenic phage. Additional factors influencing the proportion of toxigenic/non-toxigenic bacteria might be zoonotic maintenance, which might favor the emergence of toxigenic species by an unknown mechanism or the more moderate toxin expression in C. ulcerans which might be favorable for better host adaption than higher toxin levels such as produced by C. diphtheriae.

\section{Conclusions}

We prove the hypothesis that $C$. ulcerans is transmitted by a zoonotic pathway based on molecular data using a whole genome sequencing approach. To better understand the virulence potential of $C$. ulcerans, we inspected genome sequencing data for possible events of horizontal gene transfer which could cause increased virulence of $C$. ulcerans strains. We show that acquisition of virulence 
factors can take place very rapidly, as demonstrated by a phage integration event carrying a putative virulence factor, similar to a virulence factor known from S. enterica. This finding illustrates the importance of methods such as NGS in epidemiology, which can detect novel gene acquisitions, which can have a high impact on the virulence of pathogens. Additionally, we identified a novel, putative PAI which could potentially be subjected to horizontal gene transfer and thereby explain the high fraction of toxigenic C. ulcerans. This PAI is, to our knowledge, the first example of a DT gene locus not associated with a prophage and will be very important for understanding the pathogenesis of diphtheria-like disease caused by $C$. ulcerans. For the future it will be crucial to analyze this novel, putative DT transmission pathway in molecular detail to understand the emerging pathogen $C$. ulcerans.

\section{Additional file}

Additional file 1: Figure S1. Phylogenetic analysis of whole genome sequencing (WGS) and MLST data with additional methods: maximum likelihood, maximum parsimony and neighborhood joining algorithms.

\section{Abbreviations}

DT: diphtheria toxin; HO: homeodomain; HTH: helix-turn-helix; MLST: multilocus sequence typing; NCLoD: National Consiliary Laboratory on Diphtheria; NGS: next generation sequencing; PCR: polymerase chain reaction;

PAl: pathogenicity island; SNP: single nucleotide polymorphism.

\section{Competing interests}

The authors declare that they have no competing interests.

\section{Authors' contributions}

DMM, GM, HB and AS conceived experiments. DMM, GM and SK performed NGS data acquisition and analysis. RK performed micro- and molecular biological analysis. DMM and AS wrote the manuscript. All authors contributed, read and approved the final manuscript.

\section{Acknowledgements}

We thank Wolfgang Schmidt for the cultivation and microbiological characterization of the Corynebacteria, and Cecilia Hizo-Teufel for help with the DNA preparation. The study was partly supported by the Bavarian State Ministry of Health and Care (Project 13-30) and by the German Federal Ministry of Health via the Robert Koch-Institute and its National Reference Laboratories Network (09-47, FKZ 1369-359 and FKZ 415). The funders had no role in study design, data collection and analysis, decision to publish, or preparation of the manuscript.

\section{Author details}

${ }^{1}$ LGL, Bavarian Health and Food Safety Authority, Oberschleißheim 85764 Germany. ${ }^{2}$ National Consiliary Laboratory on Diphtheria, Oberschleißheim 85764, Germany. ${ }^{3}$ Laboratory for Functional Genome Analysis, Gene Center, Ludwig-Maximilians-University Munich, Munich 81377, Germany.

Received: 15 July 2014 Accepted: 17 November 2014

Published online: 28 November 2014

\section{References}

1. Funke G, von Graevenitz A, Clarridge JE 3rd, Bernard KA: Clinical microbiology of coryneform bacteria. Clin Microbiol Rev 1997, 10:125-159.

2. Wagner KS, White JM, Crowcroft NS, De Martin S, Mann G, Efstratiou A: Diphtheria in the United Kingdom, 1986-2008: the increasing role of Corynebacterium ulcerans. Epidemiol Infect 2010, 138:1519-1530.
3. Bonmarin I, Guiso N, Le Fleche-Mateos A, Patey O, Patrick AD, Levy-Bruhl D: Diphtheria: a zoonotic disease in France? Vaccine 2009, 27:4196-4200.

4. Tiwari TS, Golaz A, Yu DT, Ehresmann KR, Jones TF, Hill HE, Cassiday PK, Pawloski LC, Moran JS, Popovic T, Wharton M: Investigations of 2 cases of diphtheria-like illness due to toxigenic Corynebacterium ulcerans. Clin Infect Dis 2008, 46:395-401.

5. Sing A: Zur Charakterisierung von C.-diphtheriae-verdächtigen Isolaten. German: Robert Koch-Institut Epid Bull; 2008:23-25.

6. Berger A, Huber I, Merbecks SS, Ehrhard I, Konrad R, Hormansdorfer S, Hogardt M, Sing A: Toxigenic Corynebacterium ulcerans in woman and cat. Emerg Infect Dis 2011, 17:1767-1769. doi:10.3201/eid1709.110391.

7. Corti MA, Bloemberg GV, Borelli S, Kutzner H, Eich G, Hoelzle L, Lautenschlager S: Rare human skin infection with Corynebacterium ulcerans: transmission by a domestic cat. Infection 2012, 40:575-578.

8. Katsukawa C, Kawahara R, Inoue K, Ishii A, Yamagishi H, Kida K, Nishino S, Nagahama S, Komiya T, Iwaki M, Takahashi M: Toxigenic Corynebacterium ulcerans isolated from the domestic dog for the first time in Japan. Jpn J Infect Dis 2009, 62:171-172.

9. Hogg RA, Wessels J, Hart J, Efstratiou A, De Zoysa A, Mann G, Allen T, Pritchard GC: Possible zoonotic transmission of toxigenic Corynebacterium ulcerans from companion animals in a human case of fatal diphtheria. Vet Rec 2009, 165:691-692.

10. Schuhegger R, Schoerner C, Dlugaiczyk J, Lichtenfeld I, Trouillier A, Zeller-Peronnet V, Busch U, Berger A, Kugler R, Hormansdorfer S, Sing A: Pigs as source for toxigenic Corynebacterium ulcerans. Emerg Infect Dis 2009, 15:1314-1315. doi:10.3201/eid1508.081568.

11. Sangal V, Nieminen L, Weinhardt B, Raeside J, Tucker NP, Florea CD, Pollock KG, Hoskisson PA: Diphtheria-like disease caused by toxigenic Corynebacterium ulcerans strain. Emerg Infect Dis 2014, 20:1257-1258. doi:10.3201/eid2007.140216.

12. Hirai-Yuki A, Komiya T, Suzaki Y, Ami Y, Katsukawa C, Takahashi M, Yamamoto A, Yamada YK: Isolation and characterization of toxigenic Corynebacterium ulcerans from 2 closed colonies of cynomolgus macaques (Macaca fascicularis) in Japan. Comp Med 2013, 63:272-278.

13. Marini RP, Cassiday PK, Venezia J, Shen Z, Buckley EM, Peters Y, Taylor N, Dewhirst FE, Tondella ML, Fox JG: Corynebacterium ulcerans in ferrets. Emerg Infect Dis 2014, 20:159-161. doi:10.3201/eid2001.130675.

14. Eisenberg T, Kutzer P, Peters M, Sing A, Contzen M, Rau J: Nontoxigenic tox-bearing Corynebacterium ulcerans infection among game animals, Germany. Emerg Infect Dis 2014, 20:448-452.

15. Lartigue MF, Monnet X, Le Fleche A, Grimont PA, Benet JJ, Durrbach A, Fabre $M$, Nordmann P: Corynebacterium ulcerans in an immunocompromised patient with diphtheria and her dog. J Clin Microbiol 2005, 43:999-1001.

16. Holmes RK: Biology and molecular epidemiology of diphtheria toxin and the tox gene. J Infect Dis 2000, 181:S156-S167.

17. Choe S, Bennett MJ, Fujii G, Curmi PM, Kantardjieff KA, Collier RJ, Eisenberg D: The crystal structure of diphtheria toxin. Nature 1992, 357:216-222.

18. Naglich JG, Rolf JM, Eidels L: Expression of functional diphtheria toxin receptors on highly toxin-sensitive mouse cells that specifically bind radioiodinated toxin. Proc Natl Acad Sci U S A 1992, 89:2170-2174.

19. Naglich JG, Metherall JE, Russell DW, Eidels L: Expression cloning of a diphtheria toxin receptor: identity with a heparin-binding EGF-like growth factor precursor. Cell 1992, 69:1051-1061.

20. Bennett MJ, Eisenberg D: Refined structure of monomeric diphtheria toxin at 2.3 A resolution. Protein Sci 1994, 3:1464-1475.

21. Bell CE, Eisenberg D: Crystal structure of diphtheria toxin bound to nicotinamide adenine dinucleotide. Biochemistry 1996, 35:1137-1149.

22. Honjo T, Nishizuka Y, Hayaishi O: Adenosine diphosphoribosylation of aminoacyl transferase II by diphtheria toxin. Cold Spring Harb Symp Quant Biol 1969, 34:603-608.

23. Yamaizumi M, Mekada E, Uchida T, Okada Y: One molecule of diphtheria toxin fragment A introduced into a cell can kill the cell. Cell 1978, 15:245-250.

24. Sekizuka T, Yamamoto A, Komiya T, Kenri T, Takeuchi F, Shibayama K, Takahashi M, Kuroda M, Iwaki M: Corynebacterium ulcerans 0102 carries the gene encoding diphtheria toxin on a prophage different from the $\mathrm{C}$. diphtheriae NCTC 13129 prophage. BMC Microbiol 2012, 12:1471-2180.

25. Tao X, Schiering N, Zeng HY, Ringe D, Murphy JR: Iron, DtxR, and the regulation of diphtheria toxin expression. Mol Microbiol 1994, 14:191-197.

26. Wagner PL, Waldor MK: Bacteriophage control of bacterial virulence. Infect Immun 2002, 70:3985-3993. 
27. Schuhegger R, Lindermayer $M$, Kugler R, Heesemann J, Busch U, Sing A: Detection of toxigenic Corynebacterium diphtheriae and Corynebacterium ulcerans strains by a novel real-time PCR. J Clin Microbiol 2008, 46:2822-2823. doi:10.1128/JCM.01010-08. Epub 2008 Jun 11.; 2008.

28. Engler KH, Glushkevich T, Mazurova IK, George RC, Efstratiou A: A modified Elek test for detection of toxigenic corynebacteria in the diagnostic laboratory. J Clin Microbiol 1997, 35:495-498.

29. Goecks J, Nekrutenko A, Taylor J: Galaxy: a comprehensive approach for supporting accessible, reproducible, and transparent computational research in the life sciences. Genome Biol 2010, 11:2010-2011.

30. Blankenberg D, Von Kuster G, Coraor N, Ananda G, Lazarus R, Mangan M, Nekrutenko A, Taylor J: Galaxy: a web-based genome analysis tool for experimentalists. Curr Protoc Mol Biol 2010, 19:1-21.

31. Giardine B, Riemer C, Hardison RC, Burhans R, Elnitski L, Shah P, Zhang $Y$, Blankenberg D, Albert I, Taylor J, Miller W, Kent WJ, Nekrutenko A: Galaxy: a platform for interactive large-scale genome analysis. Genome Res 2005, 15:1451-1455

32. Luo R, Liu B, Xie Y, Li Z, Huang W, Yuan J, He G, Chen Y, Pan Q, Liu Y, Tang J, Wu G, Zhang H, Shi Y, Yu C, Wang B, Lu Y, Han C, Cheung DW, Yiu SM, Peng S, Xiaoqian Z, Liu G, Liao X, Li Y, Yang H, Wang J, Lam TW: SOAPdenovo2: an empirically improved memory-efficient short-read de novo assembler. Gigascience 2012, 1:1-18.

33. Li H, Durbin R: Fast and accurate short read alignment with BurrowsWheeler transform. Bioinformatics 2009, 25:1754-1760.

34. Trost E, Al-Dilaimi A, Papavasiliou P, Schneider J, Viehoever P, Burkovski A, Soares SC, Almeida SS, Dorella FA, Miyoshi A, Azevedo V, Schneider MP, Silva A, Santos CS, Santos LS, Sabbadini P, Dias AA, Hirata R Jr, Mattos-Guarald $A L$, Tauch A: Comparative analysis of two complete Corynebacterium ulcerans genomes and detection of candidate virulence factors. BMC Genomics 2011, 12:1471-2164.

35. Homer N, Nelson SF: Improved variant discovery through local re-alignment of short-read next-generation sequencing data using SRMA. Genome Biol 2010, 11:2010-2011.

36. Koboldt DC, Chen K, Wylie T, Larson DE, McLellan MD, Mardis ER, Weinstock GM, Wilson RK, Ding L: VarScan: variant detection in massively parallel sequencing of individual and pooled samples. Bioinformatics 2009, 25:2283-2285.

37. R Core Team: R: A Language and Environment for Statistical Computing. Vienna, Austria: R Foundation for Statistical Computing; 2014. http://www.Rproject.org/.

38. Tamura K, Stecher G, Peterson D, Filipski A, Kumar S: MEGA6: Molecular Evolutionary Genetics Analysis version 6.0. Mol Biol Evol 2013, 30:2725-2729

39. Alikhan NF, Petty NK, Ben Zakour NL, Beatson SA: BLAST Ring Image Generator (BRIG): simple prokaryote genome comparisons. BMC Genomics 2011, 12:1471-2164.

40. Rutherford K, Parkhill J, Crook J, Horsnell T, Rice P, Rajandream MA, Barrell B: Artemis: sequence visualization and annotation. Bioinformatics 2000, 16:944-945

41. Robinson JT, Thorvaldsdottir H, Winckler W, Guttman M, Lander ES, Getz G, Mesirov JP: Integrative genomics viewer. Nat Biotechnol 2011, 29:24-26.

42. Chaudhuri RR, Loman NJ, Snyder LA, Bailey CM, Stekel DJ, Pallen MJ: XBASE2: a comprehensive resource for comparative bacterial genomics. Nucleic Acids Res 2008, 36:5.

43. Darling AE, Mau B: Perna NT: progressiveMauve: multiple genome alignment with gene gain, loss and rearrangement. PLoS One 2010, 5:0011147.

44. Delcher AL, Bratke KA, Powers EC, Salzberg SL: Identifying bacterial genes and endosymbiont DNA with Glimmer. Bioinformatics 2007 23:673-679.

45. Lowe TM, Eddy SR: tRNAscan-SE: a program for improved detection of transfer RNA genes in genomic sequence. Nucleic Acids Res 1997, 25:955-964.

46. Lagesen K, Hallin P, Rodland EA, Staerfeldt HH, Rognes T, Ussery DW: RNAmmer: consistent and rapid annotation of ribosomal RNA genes. Nucleic Acids Res 2007, 35:3100-3108.

47. Altschul SF, Gish W, Miller W, Myers EW, Lipman DJ: Basic local alignment search tool. J Mol Biol 1990, 215:403-410.

48. Zhou Y, Liang Y, Lynch KH, Dennis JJ, Wishart DS: PHAST: a fast phage search tool. Nucleic Acids Res 2011, 39:14.
49. Silva Ado S, Barauna RA, de Sa PC, das Gracas DA, Carneiro AR, Thouvenin M, Azevedo V, Badell E, Guiso N, da Silva AL, Ramos RT: Draft genome sequence of corynebacterium ulcerans FRC58, isolated from the bronchitic aspiration of a patient in France. Genome Announc 2014, 2:01132-13.

50. Soding J, Biegert A, Lupas AN: The HHpred interactive server for protein homology detection and structure prediction. Nucleic Acids Res 2005 33:W244-W248

51. Hunter S, Jones P, Mitchell A, Apweiler R, Attwood TK, Bateman A, Bernard T, Binns D, Bork P, Burge S, de Castro E, Coggill P, Corbett M, Das U, Daugherty L, Duquenne L, Finn RD, Fraser M, Gough J, Haft D, Hulo N, Kahn D, Kelly E, Letunic I, Lonsdale D, Lopez R, Madera M, Maslen J, McAnulla C, McDowall J, et al: InterPro in 2011: new developments in the family and domain prediction database. Nucleic Acids Res 2012, 40:16.

52. Sievers F, Wilm A, Dineen D, Gibson TJ, Karplus K, Li W, Lopez R, McWilliam $H$, Remmert M, Soding J, Thompson JD, Higgins DG: Fast, scalable generation of high-quality protein multiple sequence alignments using Clustal Omega. Mol Syst Biol 2011, 7:75.

53. Waterhouse AM, Procter JB, Martin DM, Clamp M, Barton GJ: Jalview Version 2-a multiple sequence alignment editor and analysis workbench. Bioinformatics 2009, 25:1189-1191.

54. NCBI Sequence Read Archive [http://www.ncbi.nlm.nih.gov/sra]

55. Saitou N, Nei M: The neighbor-joining method: a new method for reconstructing phylogenetic trees. Mol Biol Evol 1987, 4:406-425.

56. Efron B, Halloran E, Holmes S: Bootstrap confidence levels for phylogenetic trees. Proc Natl Acad Sci U S A 1996, 93:7085-7090.

57. Konig C, Meinel DM, Margos G, Konrad R, Sing A: Multilocus Sequence Typing of Corynebacterium ulcerans Provides Evidence for Zoonotic Transmission and for Increased Prevalence of Certain Sequence Types among Toxigenic Strains. J Clin Microbiol 2014, 52:4318-4324.

58. Brussow H, Canchaya C, Hardt WD: Phages and the evolution of bacterial pathogens: from genomic rearrangements to lysogenic conversion. Microbiol Mol Biol Rev 2004, 68:560-602.

59. Tenor JL, McCormick BA, Ausubel FM, Aballay A: Caenorhabditis elegans-based screen identifies Salmonella virulence factors required for conserved host-pathogen interactions. Curr Biol 2004, 14:1018-1024.

60. Cerdeno-Tarraga AM, Efstratiou A, Dover LG, Holden MT, Pallen M, Bentley SD Besra GS, Churcher C, James KD, De Zoysa A, Chillingworth T, Cronin A, Dowd L, Feltwell T, Hamlin N, Holroyd S, Jagels K, Moule S, Quail MA, Rabbinowitsch E, Rutherford KM, Thomson NR, Unwin L, Whitehead S, Barrell BG, Parkhill J: The complete genome sequence and analysis of Corynebacterium diphtheriae NCTC13129. Nucleic Acids Res 2003, 31:6516-6523.

61. Iwaki M, Komiya T, Yamamoto A, Ishiwa A, Nagata N, Arakawa Y, Takahashi M: Genome organization and pathogenicity of Corynebacterium diphtheriae C7(-) and PW8 strains. Infect Immun 2010, 78:3791-3800.

62. Trost E, Blom J, Soares Sde C, Huang IH, Al-Dilaimi A, Schroder J, Jaenicke S, Dorella FA, Rocha FS, Miyoshi A, Azevedo V, Schneider MP, Silva A, Camello TC, Sabbadini PS, Santos CS, Santos LS, Hirata R, Mattos-Guaraldi AL, Efstratiou A, Schmitt MP, Ton-That H, Tauch A: Pangenomic study of Corynebacterium diphtheriae that provides insights into the genomic diversity of pathogenic isolates from cases of classical diphtheria, endocarditis, and pneumonia. J Bacteriol 2012, 194:3199-3215.

63. Cianciotto N, Serwold-Davis T, Groman N, Ratti G, Rappuoli R: DNA sequence homology between attB-related sites of Corynebacterium diphtheriae, Corynebacterium ulcerans, Corynebacterium glutamicum, and the attP site of gamma-corynephage. FEMS Microbiol Lett 1990, 54:299-301.

64. Bose B, Auchtung JM, Lee CA, Grossman AD: A conserved anti-repressor controls horizontal gene transfer by proteolysis. Mol Microbiol 2008, 70:570-582.

65. Mannervik M: Target genes of homeodomain proteins. Bioessays 1999, 21:267-270.

66. Hacker J, Kaper JB: Pathogenicity islands and the evolution of microbes. Annu Rev Microbiol 2000, 54:641-679.

67. Sing A, Hogardt M, Bierschenk S, Heesemann J: Detection of differences in the nucleotide and amino acid sequences of diphtheria toxin from Corynebacterium diphtheriae and Corynebacterium ulcerans causing extrapharyngeal infections. J Clin Microbio/ 2003, 41:4848-4851. 
68. Feng $Y, X u$ HF, Li QH, Zhang SY, Wang $C X$, Zhu DL, Cao FL, Li YG, Johnston RN, Zhou J, Liu GR, Liu SL: Complete genome sequence of Salmonella enterica serovar pullorum RKS5078. J Bacteriol 2012, 194:06507-06511.

69. Ott L, McKenzie A, Baltazar MT, Britting S, Bischof A, Burkovski A, Hoskisson PA: Evaluation of invertebrate infection models for pathogenic corynebacteria. FEMS Immunol Med Microbiol 2012, 65:413-421.

doi:10.1186/s13073-014-0113-3

Cite this article as: Meinel et al: Next generation sequencing analysis of nine Corynebacterium ulcerans isolates reveals zoonotic transmission and a novel putative diphtheria toxin-encoding pathogenicity island. Genome Medicine 2014 6:113.

\section{Submit your next manuscript to BioMed Central and take full advantage of:}

- Convenient online submission

- Thorough peer review

- No space constraints or color figure charges

- Immediate publication on acceptance

- Inclusion in PubMed, CAS, Scopus and Google Scholar

- Research which is freely available for redistribution 